\title{
Vitamin K2 Suppresses Proliferation and Motility of Hepatocellular Carcinoma Cells by Activating Steroid and Xenobiotic Receptor
}

\author{
Kotaro AZUMA*, TOMOHIKo URANO*,**, YASUYOSHI OUCHI* AND SATOSHI INOUE*,**,*** \\ * Department of Geriatric Medicine, Graduate School of Medicine, The University of Tokyo, Tokyo 113-8655, Japan \\ ** Department of Anti-Aging Medicine, Graduate School of Medicine, The University of Tokyo, Tokyo 113-8655, Japan \\ *** Division of Gene Regulation and Signal Transduction, Research Center for Genomic Medicine, Saitama Medical University, Saitama \\ 350-1241, Japan
}

\begin{abstract}
Vitamin K2, known as a cofactor for $\gamma$-carboxylase, also serves as a ligand of a nuclear receptor, Steroid and Xenobiotic Receptor (SXR). Several clinical trials revealed that vitamin K2 reduced de novo formation and recurrence of hepatocellular carcinoma (HCC). To examine the role of SXR in HCC as a receptor activated by vitamin K2, the cells stably overexpressing SXR were established using a HCC cell line, HuH7. Overexpression of SXR resulted in reduced proliferation and motility of the cells. Further suppression of proliferation and motility was observed when SXR overexpressing clones were treated with vitamin $\mathrm{K} 2$. These results suggest that the activation of SXR could contribute to tumor suppressive effects of vitamin K2 on HCC cells.
\end{abstract}

Key words: Vitamin K2, Hepatocellular carcinoma, Steroid and xenobiotic receptor

(Endocrine Journal 56: 843-849, 2009)

VITAMIN $\mathrm{K}$ is a fat soluble vitamin which is involved in blood coagulation and bone metabolism. Naturally existing vitamin $\mathrm{K}$ includes vitamin K1 (phylloquinone) and vitamin K2 (menaquinone). Phylloquinone is contained mainly in vegetables, while menaquinone is synthesized by microorganism and is contained in food such as natto (fermented soy beans). Menatetrenone (MK-4) is one of the forms of vitamin K2 which has shown to have effect on bone fracture prevention $[1,2]$. In Japan, Korea and Thailand, MK-4 is clinically used for treatment of osteoporosis.

A recent clinical trial revealed that administration of MK-4 prevented the occurrence of hepatocellu-

Received Apr. 9, 2009; Accepted Jun. 1, 2009 as K09E-108 Released online in J-STAGE as advance publication Jun. 24, 2009

Correspondence to: Satoshi INOUE, M.D., Ph.D., Department of Geriatric Medicine, Graduate School of Medicine, The University of Tokyo, 7-3-1 Hongo, Bunkyo-ku, Tokyo 113-8655, Japan.

E-mail: INOUE-GER@h.u-tokyo.ac.jp

The abbreviations used are: DMEM, Dulbecco's modified Eagle's medium; FCS, fetal calf serum; HCC, hepatocellular carcinoma; SXR, Steroid and Xenobiotic Receptor lar carcinoma (HCC) in patients of liver cirrhosis [3]. Moreover, in another clinical trials, MK-4 delayed the recurrence of $\mathrm{HCC}$ after treatment $[4,5]$.

Vitamin $\mathrm{K} 2$ is known as a co-enzyme for $\gamma$-carboxylase. Some of coagulation factors are substrate of $\gamma$-carboxylase, and $\gamma$-carboxylation of such coagulation factors is necessary to maintain their activity. Also in the bone tissue, some proteins such as osteocalcin [6] and matrix Gla protein [7] require $\gamma$-carboxylation.

Recently we found that vitamin K2 also functions as a ligand for Steroid and Xenobiotic Receptor (SXR) [8]. SXR, also known as PXR, PAR, and NR1I2, is a nuclear receptor expressed mainly in liver and small intestine [9]. SXR belongs to orphan nuclear receptor family, but now physiological ligands are found to be secondary bile acids, such as lithocholic acid [10]. Some chemical compounds, such as rifampicin, phenobarbital and paclitaxel also function as ligands for SXR [11, 12]. SXR forms heterodimer with RXR on ligand stimulus, and binds SXR responsive element in promoter or enhancer of target genes [9]. Cyp3A4, 
UGT1A1, and MDR1, which are involved in drug metabolism and excretion, are known as SXR target genes reflecting its function as a xenobiotic sensor [9]. We showed vitamin K2 binds to SXR and induced SXR target genes [8]. Moreover, tsukushi, one of the vitamin K2-depenent SXR target genes in osteoblasts was involved in collagen accumulation, suggesting that vitamin $\mathrm{K} 2$ contributes to bone strength partially through the activation of SXR [13].

In the present study, we hypothesized vitamin $\mathrm{K} 2$ improves prognosis of HCC through the activation of SXR. Therefore, we analyzed the effects of SXR overexpression and stimulation with vitamin $\mathrm{K} 2$ on the HCC cells. We here demonstrate vitamin K2 suppressed proliferation and motility of $\mathrm{HuH} 7 \mathrm{HCC}$ cells, and these effects required the expression of SXR.

\section{Materials and Methods}

\section{Plasmids}

FLAG-tagged human SXR (FLAG-SXR) and tk(Cyp3A4) - $_{3}$-Luc containing three-copy SXR responsive elements from human cytochrome P-450 (Cyp) 3A4 promoter were described previously [13]. FLAG-HAtagged human SXR was generated by site directed mutagenesis using primers (forward: 5'-p-GAGGTGA GACCCAAAGAAAGCTGG-3', reverse: 5'-CTCTA GACCTCCGGCGTAGTCGGGCACGTCGTAGGGG TATCCTCCATCGAGCTTGT-3') and FLAG-SXR as a template.

\section{Cell culture and transfection}

HuH7 is obtained from RIKEN Cell Bank (Tsukuba, Japan). HepG2 and LS180 were purchased from American Type Culture Collection (ATCC) (Manassas, VA, USA). HuH7, HepG2, and LS180 cells were maintained in Dulbecco's modified Eagle's medium (DMEM) with $10 \%$ fetal calf serum (FCS), at $37^{\circ} \mathrm{C}$ under $5 \% \mathrm{CO}_{2}$. $\mathrm{HuH} 7$ and HepG2 cells were cultured in phenol red-free DMEM with 5\% charcoal/dextrantreated FCS for at least $24 \mathrm{~h}$ prior to rifampicin (Nakalai Tesque, Kyoto, Japan), MK-4 (gifted by Eisai Co., Ltd., Tokyo, Japan), or vehicle $(0.1 \%$ ethanol) treatment. Transfection was performed using FuGENE 6 (Roche, Castle Hill, Australia). To establish stable transfectants, $\mathrm{HuH} 7$ clones were selected using G418 (SIGMA, St.
Louis, MO, USA) at a concentration of $250 \mu \mathrm{g} / \mathrm{mL}$.

\section{Reverse-transcription PCR analysis}

Total RNA was isolated using the TOTALLY RNA kit (Ambion, Austin, TX, USA). First strand cDNA was generated by using PrimeScript RT reagent Kit (TAKARA, Kyoto, Japan). For PCR amplification, the primer sequences are as follows: human SXR (forward: 5'-CCTTTGACACTACCTTCTCC-3', reverse: 5'GTTGACACAGCTCGAAAGCG-3'); human 18S rRNA: (forward: 5'-AAGCATTTGCCAAGAATGTTTT -3', reverse: 5'-TTAAGTTTCAGCTTTGCAACCA-3'). mRNAs were quantified by real-time PCR using SYBR green PCR master mix (Applied Biosystems, Foster City, CA, USA) and the 7500 Fast Real-Time PCR system (Applied Biosystems). The evaluation of relative differences of PCR product amounts among the treatment groups was carried out using $18 \mathrm{~S}$ rRNA as an internal control.

\section{Luciferase Assay}

Luciferase assay was performed using $\mathrm{HuH7}$ cells $\left(1 \times 10^{4}\right.$ cells/well on 24 -well plates) transfected with $200 \mathrm{ng}$ of tk-(Cyp3A4) ${ }_{3}$-Luc, $200 \mathrm{ng}$ of pRL-CMV (Promega, Madison, WI, USA), and 200 ng of FLAGHA-pcDNA3 or FLAG-HA-tagged SXR plasmids using the FuGENE 6 reagent (Roche). When ligand stimulation was done, cells were treated with $10 \mu \mathrm{M}$ rifampicin (Nakalai Tesque), MK-4 (Eisai Co., Ltd.), or vehicle $(0.1 \%$ ethanol) for $1 \mathrm{~h}$ prior to transfection. Luciferase activities were determined by a Mithras LB 940 microplate luminometer (Berthold Technologies; Bad Wilbad, Germany) using the dual-luciferase assay system (Promega). Firefly luciferase activity was normalized to Renilla luciferase, which was used as a transfection control.

\section{Cell proliferation assay}

Cells were seeded in 96-well plates at a density of 1,000 cells/well. The viable cell number was quantified using tetrazolium salt, WST-8 (Nakalai Tesque) that could be converted to a water-soluble formazan by metabolically active cells. Spectrophotometric absorbance for formazan dye was measured at $450 \mathrm{~nm}$, with absorbance at $655 \mathrm{~nm}$ as reference using microplate reader (BIO-RAD, Hercules, CA, USA). 


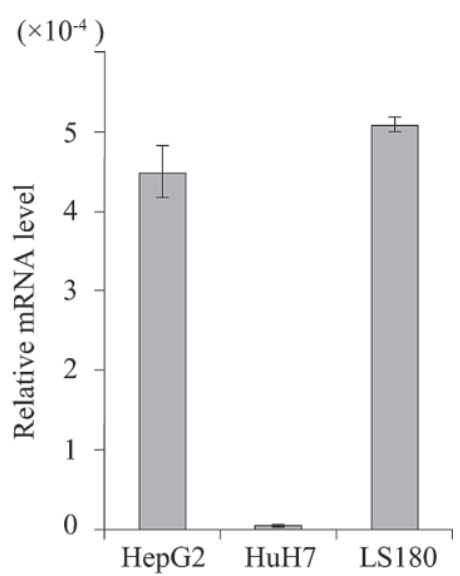

Fig. 1. Expression of SXR in hepatocellular carcinoma cell lines. SXR mRNA was evaluated by quantitative realtime PCR. Expression in a colorectal carcinoma cell line, LS 180, is shown as a positive control. Bars represent the mean value of relative mRNA level $\pm \operatorname{SEM}(n=2)$.

\section{Cell migration assay}

The cell migration assay was performed as previously described [14]. Briefly, the number of $\mathrm{HuH7}$ cells migrating through a polyethylene terephthalate (PET) filter with $8-\mu \mathrm{m}$ pores (Beckton Dickinson, Franklin Lake, NJ, USA) in $24 \mathrm{~h}$ was counted under microscopic examination.

\section{Statistical analyses}

Differences between the mean values were analyzed using the Student's $t$-test.

\section{Results}

Detection of endogenous expression of SXR in HCC cell lines

To test the hypothesis that vitamin K2 affect the character of HCC cells by regulating SXR induced genes, the expression of SXR in HCC cells were evaluated by Reverse Transcription-PCR analysis. Messenger RNA of SXR was detected in HepG2 and $\mathrm{HuH7}$, HCC cells, though expression level of SXR in HuH7 was relatively low (Fig. 1). Expression level of SXR mRNA in a colorectal carcinoma cell line, LS180,

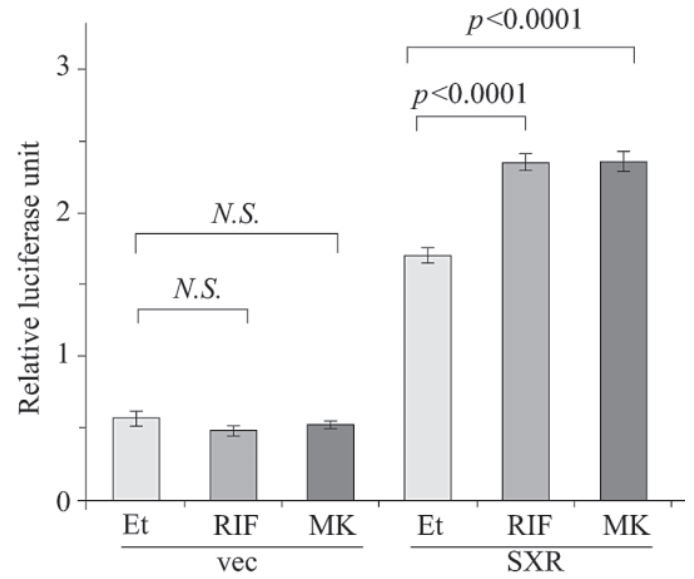

Fig. 2. Exogenous SXR was functional in $\mathrm{HuH7}$ cells. $\mathrm{HuH7}$ cells were transfected with DNA mixture of tk-

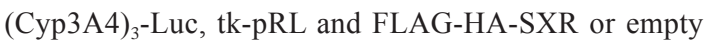
vector. These cells were treated with rifampicin (RIF) $(10 \mu \mathrm{M}), \mathrm{MK}-4(10 \mu \mathrm{M})$ or ethanol (vehicle) for $24 \mathrm{~h}$ and promoter activity was evaluated. Bars represent the mean value of relative luciferase unit $\pm \operatorname{SEM}(\mathrm{n}=8)$.

is shown as a positive control. These results indicate that endogenous SXR is expressed in HCC cell lines, like in some of colorectal carcinoma cell lines, though the level of expression varies among cell lines.

Ligand dependent promoter activation of exogenous SXR in HuH7 cells

To analyze SXR function in HCC cells, HuH7 cells, which have relatively low expression of SXR were transfected with SXR expression vector and the effect of vitamin K2 (Menatetrenone; MK-4) was evaluated with dual luciferase reporter assay. The effect of SXR agonist, rifampicin (RIF) was also examined. SXR responsive element in the promoter of Cyp3 44 gene, a known SXR target gene, was used as reporter plasmid. In SXR transfected cells, basal activity of promoter was increased even with vehicle stimulation (Fig. 2). The significant enhancement of promoter activity was observed with rifampicin or MK-4 stimulation in SXR transfected cells (Fig. 2). Activation of endogenous SXR was not detected in this method (Fig. 2).

\section{Growth suppression in SXR overexpressing cells}

To examine the role of SXR in HCC cells, the clone stably overexpressing SXR was established. Relative expression of SXR in the clone overexpressing exog- 
A

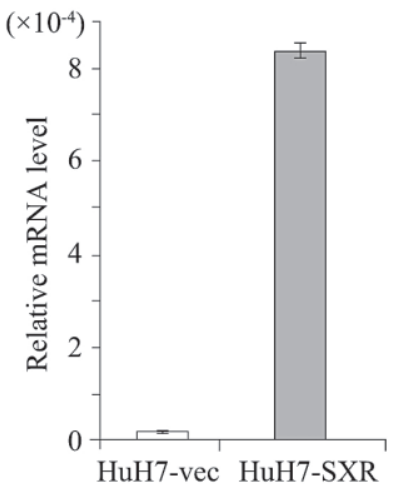

B

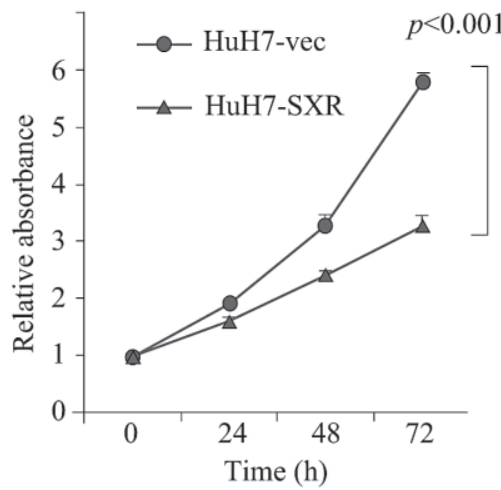

C

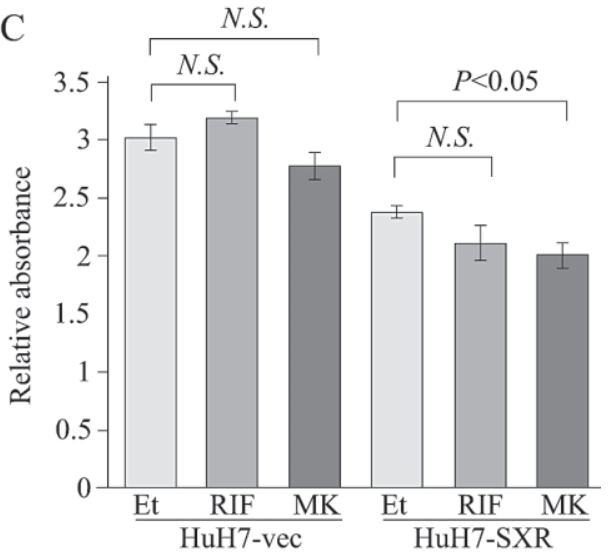

Fig. 3. Vitamin K2 dependent growth suppression in the SXR overexpressing HuH7 cells. (A) SXR was stably expressed in HuH7 cells. Expression levels of SXR mRNA in the SXR overexpressing clone (HuH7-SXR) and the control clone (HuH7-vec) were measured by quantitative real-time PCR. Bars represent the mean value of relative mRNA level \pm SEM ( $n=3$ ). (B) Proliferation of SXR overexpressing cells was suppressed. HuH7-SXR and HuH-vec cells were seeded at a density of 1,000 cells/well and cultured in DMEM with $10 \%$ FCS. The cell growth was assayed using WST-8 tetrazolium salt. Mean \pm SEM of relative absorbance at $450 \mathrm{~nm}$ for each clone on each day normalized to values at day 0 was shown $(\mathrm{n}=3)$. (C) Ligand dependent growth suppression in the SXR overexpressing cells. HuH7-SXR and HuH7-vec cells were seeded at a density of 1,000 cells/well and cultured in phenol red free DMEM with charcoal/dextran-treated FCS (5\%) with rifampicin (RIF) $(10 \mu \mathrm{M})$, MK-4 (10 $\mu \mathrm{M})$ or ethanol (Et). The cell growth was assayed using WST- 8 tetrazolium salt. Bar represents the mean \pm SEM of relative absorbance at $450 \mathrm{~nm}$ for each clone on day 4 normalized to values at day $0(\mathrm{n}=4)$. N.S., not significant.

enous SXR (HuH7-SXR) and vector clone (HuH7-vec) was confirmed by quantitative real-time PCR (Fig. 3A).

Then, the effect of SXR expression on cell proliferation was evaluated. The SXR overexpressing $\mathrm{HuH} 7$ clone (HuH7-SXR) and the control clone (HuH7-vec) were seeded at a density of 1000 cells/well and cultured in DMEM with 10\% FCS. The cell growth was assayed using WST-8 tetrazolium salt in every 24 hours. The proliferation of $\mathrm{HuH7}$ cells was significantly suppressed in SXR overexpressing clones (Fig. 3B).

Next, the effects of SXR ligands were tested. SXR overexpressing clone (HuH7-SXR) and the control clone (HuH7-vec) were grown for 72 hours in the presence of SXR agonist, rifampicin or MK-4 or ethanol (vehicle). Phenol red free DMEM with charcoal/ dextran-treated FCS (5\%) were used to eliminate potential ligands contained in the medium. The SXR overexpressing clone showed slower growth rate than the vector clone. Presence of rifampicin or MK-4 further suppressed the proliferation in the SXR overexpressing clone, and the suppression with MK-4 treatment was statistically significant (Fig 3C). In the vector clone, growth suppressive effect of rifampicin was absent and slight growth suppression with MK-4 treatment was not significant (Fig. 3C).

\section{Suppression of motility of SXR overexpressing cells}

We then focused on the effects of SXR on motility of $\mathrm{HuH} 7$ cells. The SXR overexpressing clone (HuH7SXR) and the control clone (HuH7-vec) were seeded on collagen coated filter with $8 \mu \mathrm{m}$ pores in DMEM with $10 \% \mathrm{FCS}$, and the number of cells on the other side of the filter was counted after 24 hours. The number of migrating cells of the SXR overexpressing clone was significantly smaller than that of vector clone (Fig. $4 \mathrm{~A}$ ). Then migration assay was performed in the presence of rifampicin or MK-4. Phenol red free DMEM with charcoal/dextran-treated FCS (5\%) were used to eliminate potential ligands contained in the medium. The SXR overexpressing clone (HuH7-SXR) showed decreased motility than the vector clone (HuH7-vec) in the vehicle treatment (Fig. 4B). Stimulation of cells with rifampicin or MK-4 further suppressed migration in the SXR overexpressing clone, while rifampicin or MK-4 did not significantly affect the motility of the vector clone (Fig. 4B).

We then evaluated the effect of rifampicin and MK-4 on the motility of HepG2 cells where relatively much amount of endogenous SXR was expressed. Both of the SXR ligand, rifampicin and MK-4, sup- 
A
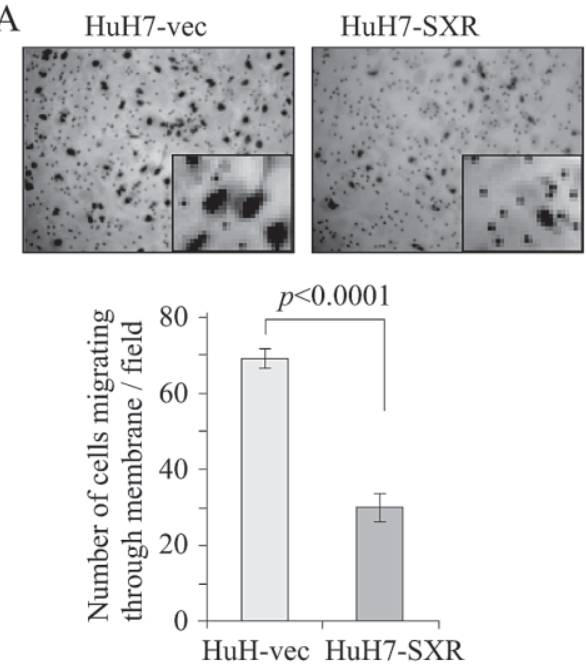

B

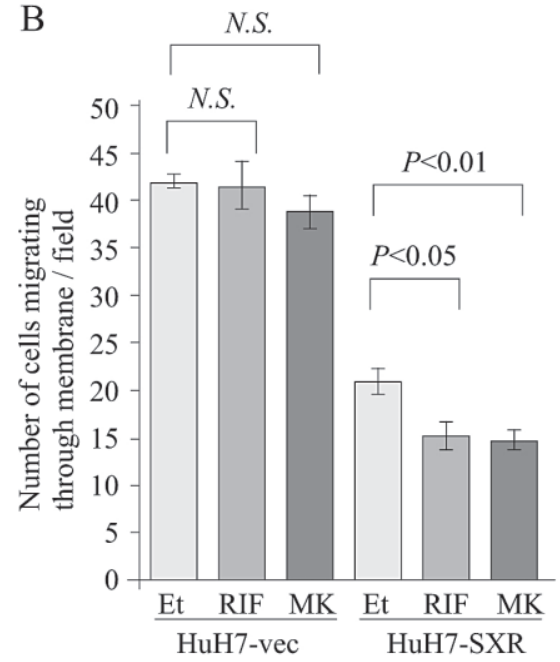

$\mathrm{C}$

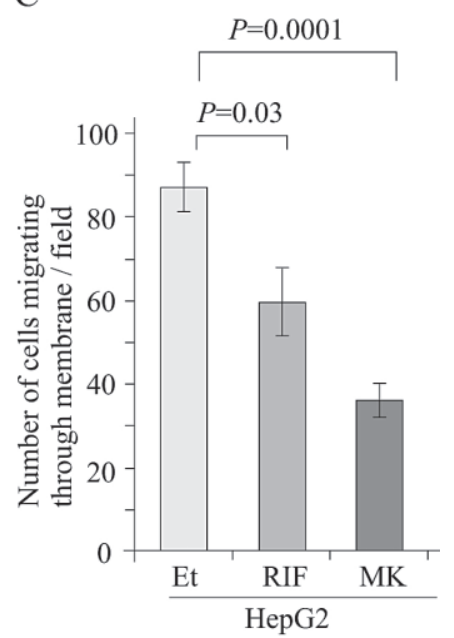

Fig. 4. Vitamin K2 dependent suppression of motility in the SXR overexpressing HuH7 cells. (A) HuH7-SXR and HuH-vec cells were seeded on the PET filter with 8 - $\mu$ m pores and migration activity was evaluated in the DMEM with $10 \%$ FCS. Number of cells migrating through a PET filter in $24 \mathrm{~h}$ was counted. Upper panels; cells on the lower side of the filters were stained with Giemsa's staining solution and visualized under a microscope. Representative views used to count the cells are shown at a 200x magnification. Magnified views of membrane pores and migrating cells are shown in the insets. Lower graph; bars represent the mean number of cells \pm SEM counted in five fields. (B) Ligand dependent suppression of motility in the SXR overexpressing HuH7 cells. HuH7-SXR and HuH7-vec cells were seeded on the PET filter with 8- $\mu$ m pores and migration activity was evaluated in phenol red free DMEM with charcoal/dextran-treated FCS $(5 \%)$ with rifampicin (RIF) $(10 \mu \mathrm{M}), \mathrm{MK}-4(10 \mu \mathrm{M})$ or ethanol (Et). Bars represent the mean number of cells \pm SEM counted in five fields. N.S., not significant. (C) Ligand dependent suppression of motility in HepG2 cells. HepG2 cells were seeded on the PET filter with 8 - $\mu$ m pores and migration activity was evaluated in phenol red free DMEM with charcoal/dextran-treated FCS (5\%) with rifampicin (RIF) $(10 \mu \mathrm{M}), \mathrm{MK}-4$ (10 $\mu \mathrm{M})$ or ethanol (Et). Bars represent the mean number of cells \pm SEM counted in five fields.

pressed the motility of HepG2 significantly (Fig. 4C).

\section{Discussion}

In the present study, we showed expression and activation of steroid and xenobiotic receptor SXR suppressed proliferation and motility of hepatocellular carcinoma HuH7 cells. Our results support the hypothesis that a novel mechanism of vitamin $\mathrm{K} 2$, activation of SXR, contributes to efficacy of first and second prevention of HCC.

Liver is one of the organ in which SXR is mainly expressed. However, whether SXR is expressed in HCC cells has not been studied so far. We demonstrated in this study that expression of SXR was detectable by RT-PCR and that the level of expression varied among cell lines. Considering HCC arises from hepatocytes, it is reasonable that some $\mathrm{HCC}$ cells inherit expression pattern of hepatocytes. The activity of endogenous SXR was not detected in $\mathrm{HuH7}$ cells by luciferase as- say using tk-(Cyp3A4) $)_{3}$-Luc. Together with the results of vector clones in growth assay and motility assay, amount of SXR molecules in HuH7 cells we used may be not enough to cause visible effect. HepG2 cells, in which we could detect higher amount of endogenous SXR mRNA compared with HuH7 cells (Fig. 1), showed suppressed motility with SXR ligand stimulation. We could speculate that the response to MK-4 in HCC cells may depend on the expression level of SXR. This result also suggested that effects of MK-4 through SXR activation can be observed in HCC cells other than $\mathrm{HuH} 7$ cells.

In our study, we employed the MK-4 concentration mainly at $10 \mu \mathrm{M}$. Plasma concentration of single administration of pharmacological dose $(15 \mathrm{mg})$ of menatetrenone in healthy male volunteers was reported to be approximately $1 \mu \mathrm{M}$ [15]. In the experiment using rat, menatetrenone was shown to accumulate in liver at more than 10 times concentration than plasma concentration after oral administration [16]. Therefore, $10 \mu \mathrm{M}$ is considered to be an adequate concentration 
to evaluate pharmacological effect of menatetrenone.

As for the mechanism of anti-tumor effect of vitamin K2, several theories were proposed. Otsuka et al. reported that vitamin $\mathrm{K} 2$ inhibited the growth and motility of HCC by activation of protein kinase A and by inhibition of Rho kinase [17]. Suzuki et al. showed Des-gamma-carboxyl prothrombin (DCP), also known as PIVKAII, induced proliferation of HCC [18]. Some of the mechanisms are explained by the function of vitamin $\mathrm{K} 2$ as a co-enzyme for $\gamma$-carboxylase. Besides these mechanisms of vitamin K2 action, we propose another mechanism through the activation of SXR. Considering the weaker effect of vitamin K2 on the vector clone compared with SXR overexpressing clone in the present study, we assume that vitamin $\mathrm{K} 2$ affects growth and motility of HCC mainly by activating SXR in some conditions. This notion is further supported by the results indicating rifampicin, an SXR agonist, also suppressed the motility of HuH7-SXR and HepG2. Rifampicin also tended to suppress the growth of HuH7-SXR, though this effect was not statistically significant. We assume, unlike vitamin K2 specific $\gamma$-carboxylase dependent mechanism, SXR dependent mechanism is not specific for vitamin K2 and other potential ligands can trigger this effect.

As for the downstream of SXR, we consider that the function of SXR would be mediated by interaction with other transcription factors, or by induction of SXR responsive genes. For example, Ozaki et al. recently reported that vitamin $\mathrm{K} 2$ inhibited $\mathrm{HCC}$ growth through inhibition of NF- $\kappa B$ activation [19]. On the other hand, Zhou et al. showed mutual repressive relationship between SXR and NF- $\kappa B$ [20]. Therefore, it is tempting to speculate that the inhibition of NF- $\kappa B$ by vitamin $\mathrm{K} 2$ could be mediated by activation of SXR.
It is noteworthy that function of SXR might be regulated not only by its ligands. Our result of luciferase assay showed that promoter activity was elevated only with SXR expression even in the absence of ligands and further enhanced with ligand treatment. In the present study, phenol red-free DMEM and charcoal/ dextran-treated FCS were used to minimize the effect of potential ligands contained in the medium. Although suppression of proliferation and motility in HuH-SXR compared with $\mathrm{HuH}-\mathrm{vec}$ even without ligand stimulation can be due to residual ligands in the medium, it is also possible that other factors such as modification of SXR molecule affect the transcriptional activity or interaction with other transcription factors.

In conclusion, we showed that activation of SXR suppresses proliferation and motility of HCC cells. These results suggest that vitamin K2 prevents occurrence and recurrence of HCC not only by activating $\gamma$-carboxylase pathway but also by a novel mechanism, activating SXR. Interacting molecules and target genes of SXR should be clarified to understand detailed mechanism of SXR functions and to find novel therapeutic targets of HCC.

\section{Acknowledgements}

Grant support: Genome Network Project and DECODE from the Ministry of Education, Culture, Sports, Science and Technology, Grants from the Japan Society for the Promotion of Science, and Grants-in-Aid from the Ministry of Health, Labor and Welfare. We are grateful to Mr. A. Okada for his technical assistance.

\section{References}

1. Shiraki M, Shiraki Y, Aoki C, Miura M (2000) Vitamin K2 (menatetrenone) effectively prevents fractures and sustains lumbar bone mineral density in osteoporosis. $J$ Bone Miner Res 15: 515-521.

2. Cockayne S, Adamson J, Lanham-New S, Shearer MJ, Gilbody S, Torgerson DJ (2006) Vitamin K and the prevention of fractures: systematic review and metaanalysis of randomized controlled trials. Arch Intern Med 166: 1256-1261.

3. Habu D, Shiomi S, Tamori A, Takeda T, Tanaka T, Kubo S, Nishiguchi S (2004) Role of vitamin K2 in the development of hepatocellular carcinoma in women with viral cirrhosis of the liver. JAMA 292: 358-361.

4. Mizuta T, Ozaki I, Eguchi Y, Yasutake T, Kawazoe S, Fujimoto K, Yamamoto K (2006) The effect of menatetrenone, a vitamin $\mathrm{K} 2$ analog, on disease recurrence and survival in patients with hepatocellular carcinoma after curative treatment: a pilot study. Cancer 106:867872.

5. Kakizaki S, Sohara N, Sato K, Suzuki H, Yanagisawa M, Nakajima H, Takagi H, Naganuma A, Otsuka T, Takahashi H, Hamada T, Mori M (2007) Preventive ef- 
fects of vitamin $\mathrm{K}$ on recurrent disease in patients with hepatocellular carcinoma arising from hepatitis $\mathrm{C}$ viral infection. J Gastroenterol Hepatol 22: 518-522.

6. Price P A, Otsuka A A, Poser J W, Kristaponis J, Raman N (1976) Characterization of a gamma-carboxyglutamic acid-containing protein from bone. Proc Natl Acad Sci U S A 73:1447-1451.

7. Luo G, Ducy P, McKee MD, Pinero GJ, Loyer E, Behringer RR, Karsenty G (1997) Spontaneous calcification of arteries and cartilage in mice lacking matrix GLA protein. Nature 386: 78-81.

8. Tabb MM, Sun A, Zhou C, Grün F, Errandi J, Romero K, Pham H, Inoue S, Mallick S, Lin M, Forman BM, Blumberg B (2003) Vitamin K2 regulation of bone homeostasis is mediated by the steroid and xenobiotic receptor SXR. J Biol Chem 278: 43919-439.

9. Blumberg B, Sabbagh W Jr, Juguilon H, Bolado J Jr, van Meter CM, Ong ES, Evans RM (1998) SXR, a novel steroid and xenobiotic-sensing nuclear receptor. Genes Dev 12: 3195-3205.

10. Xie W, Radominska-Pandya A, Shi Y, Simon CM, Nelson MC, Ong ES, Waxman DJ, Evans RM (2001) An essential role for nuclear receptors SXR/PXR in detoxification of cholestatic bile acids. Proc Natl Acad Sci U S A 98: 3375-3380.

11. Xie W, Barwick JL, Downes M, Blumberg B, Simon CM, Nelson MC, Neuschwander-Tetri BA, Brunt EM, Guzelian PS, Evans RM (2000) Humanized xenobiotic response in mice expressing nuclear receptor SXR. Nature 406: 435-439.

12. Synold TW, Dussault I, Forman BM (2001) The orphan nuclear receptor SXR coordinately regulates drug metabolism and efflux. Nat Med 7: 584-590.

13. Ichikawa T, Horie-Inoue K, Ikeda K, Blumberg B, Inoue S (2006) Steroid and xenobiotic receptor SXR mediates vitamin K2-activated transcription of extracellular matrix-related genes and collagen accumu- lation in osteoblastic cells. J Biol Chem 281: 1692716934.

14. Azuma K, Tanaka M, Uekita T, Inoue S, Yokota J, Ouchi Y, Sakai R (2005) Tyrosine phosphorylation of paxillin affects the metastatic potential of human osteosarcoma. Oncogene 24: 4754-4764.

15. Uematsu T, Nagashima S, Niwa M, Kohno K, Sassa T, Ishii M, Tomono Y, Yamato C, Kanamaru M (1996) Effect of dietary fat content on oral bioavailability of menatetrenone in humans. J Pharm Sci 85: 1012-1016.

16. Sano Y, Tadano K, Kaneko K, Kikuchi K, Yuzuriha T (1995) Distribution of menaquinone-4, a therapeutic agent for osteoporosis, in bone and other tissues of rats. J Nutr Sci Vitaminol 41: 499-514.

17. Otsuka M, Kato N, Shao RX, Hoshida Y, Ijichi H, Koike Y, Taniguchi H, Moriyama M, Shiratori Y, Kawabe T, Omata M (2004) Vitamin K2 inhibits the growth and invasiveness of hepatocellular carcinoma cells via protein kinase A activation. Hepatology 40: 243-251.

18. Suzuki M, Shiraha H, Fujikawa T, Takaoka N, Ueda N, Nakanishi Y, Koike K, Takaki A, Shiratori Y (2005) Des-gamma-carboxy prothrombin is a potential autologous growth factor for hepatocellular carcinoma. J Biol Chem 280: 6409-6415.

19. Ozaki I, Zhang H, Mizuta T, Ide Y, Eguchi Y, Yasutake T, Sakamaki T, Pestell RG, Yamamoto K (2007) Menatetrenone, a vitamin K2 analogue, inhibits hepatocellular carcinoma cell growth by suppressing cyclin D1 expression through inhibition of nuclear factor kappaB activation. Clin Cancer Res 13: 2236-2245.

20. Zhou C, Tabb MM, Nelson EL, Grün F, Verma S, Sadatrafiei A, Lin M, Mallick S, Forman BM, Thummel KE, Blumberg B (2006) Mutual repression between steroid and xenobiotic receptor and NFkappaB signaling pathways links xenobiotic metabolism and inflammation. J Clin Invest 116: 2280-2289. 